\title{
Simultaneous Multi-Information Fusion and Parameter Estimation for Robust 3-D Indoor Positioning Systems
}

\author{
Hui Wang, Andrei Szabo, Joachim Bamberger, and Uwe D. Hanebeck
}

\begin{abstract}
Typical WLAN based indoor positioning systems take the received signal strength (RSS) as the major information source. Due to the complicated indoor environment, the RSS measurements are hard to model and too noisy to achieve a satisfactory 3-D accuracy in multi-floor scenarios. To enhance the performance of WLAN positioning systems, extra information sources could be integrated. In this paper, a Bayesian framework is applied to fuse multi-information sources and estimate the spatial and time varying parameters simultaneously and adaptively. An application of this framework, which fuses pressure measurements, a topological building map with RSS measurements, and simultaneously estimates the pressure sensor bias, is investigated. Our experiments indicate that the localization performance is more accurate and robust by using our approach.
\end{abstract}

\section{INTRODUCTION}

Indoor positioning systems recently attracted a lot of research efforts in both academia and industry for their broad applications such as security, asset tracking, robotics, and many others [1] [2] [3]. Many promising systems utilize the received signal strength (RSS) of wireless LAN (WLAN) to infer the location information. These systems have a big advantage in the installation and maintenance cost by using the existing communication infrastructure. Numerous research results also indicate that the WLAN positioning system suffers from the noisy characteristics of radio propagation [3]. For example, in large multi-floor buildings, the location error often has a large variance due to the complexity of indoor environment and insufficient number of reachable access points (APs) [4]. Although adding more APs could improve the performance, this solution implies higher installation costs. Another solution is to integrate other location related information besides the WLAN signal. For instance, in our previous work [4] and [5], MEMS sensors were used to enhance the localization performance due to their small sizes and low prices.

To determine the user's location, the model that maps the sensed measurements (e.g, RSS, air pressure and etc.) to the location should be known. Most systems assume that this model has an accurate analytical form and it does not change with time. But in reality, the model parameters could be inaccurate and sometimes vary over time and space. For instance, the air pressure is determined not only by the

Hui Wang, Andrei Szabo, Joachim Bamberger are with Information and Communications, Corporate Technology, Siemens AG, Otto-Hahn-Ring 6, Munich, Germany. Email: \{hui.wang, andrei.szabo, joachim.bamberger\}@siemens.com

Uwe D. Hanebeck is with Intelligent Sensor-Actuator-Systems Laboratory (ISAS), Institute of Computer Science and Engineering, Universitaet Karlsruhe (TH), Karlsruhe, Germany. Email: uwe.hanebeck@ieee.org altitude but also by some unknown environmental change, which could be modeled as a spatial and time varying bias. Besides, the radio distribution should also be accurately known and it could be temporally varying due to the changes of transmission power or the movement of scatters.

This paper is intended as an investigation of using the Bayesian filtering framework to fuse location-related information sources and simultaneously estimate their unknown parameters, i.e., to solve a joint state and parameter estimation problem. Since the localization problem is usually nonlinear, the exact Bayesian filter is in general intractable. Different approximate estimators like the Extended Kalman Filter (EKF) [6], Unscented Kalman filter (UKF) [7], Particle Filter [8] or Hybrid Density Filter [9] could solve this problem. Additionally, dual estimation [10] or the expectationmaximization (EM) algorithm [11], which decouples the state and parameter estimation into two different problems in a suboptimal way, can also be used.

There already exists a class of self-estimation and calibration algorithms such as simultaneous floor identification and pressure compensation [4], simultaneous localization and learning (SLL) [3], and simultaneous localization and mapping (SLAM) [12]. These algorithms have a strong requirement with respect to initial conditions to ensure the convergence. Different from them, this paper aims to use different information sources to teach each other so that the system is ensured to be robust.

As an example application of the proposed framework, this paper simultaneously fuses RSS measurements, a discrete topological map as well as pressure measurements, and estimate the spatial and time varying pressure bias. The resulting posterior joint probabilities are proven to be in a Gaussian-mixture form. For simplicity, a dual estimation algorithm is applied, which takes a grid-based filter for location estimation and a Kalman filter for parameter estimation respectively. Our experiments in a typical multi-floor office building indicate that the location performance is more accurate and robust by using this approach even given an inaccurate initial condition.

The remainder of this paper is organized as follows: in Section II, the general multi-information fusion and parameter estimation problem is formulated. In Section III, the characteristics of the selected information sources are given. In Section IV, the specific form of the Bayesian framework is derived given the information sources in Section III. Its solution by the dual estimation is described. Section $\mathrm{V}$ presents the experiment setup and discusses the result. Finally, conclusions and an outlook to future work are given 
in Section VI.

\section{Problem Formulation}

The indoor positioning system can be modeled as a nonlinear and non-Gaussian dynamic system. We use $\boldsymbol{x}_{k}=$ $\left[x_{k}, y_{k}, z_{k}\right]^{T} \in \mathcal{L}$ to denote the 3-D location at time $k$. $\mathcal{L} \subset \mathcal{R}^{3}$ denotes the indoor location domain. $\boldsymbol{o}_{k}=$ $\left[o_{k}^{1}, \ldots, o_{k}^{N}\right]^{T} \in \mathcal{O}$ represents the sensed measurements from sensor 1 to $N$ at time $k$, including RSS measurements, pressure measurements and so on. $\mathcal{O} \subset \mathcal{R}^{N}$ denotes the oberservation domain. $\gamma_{k}=\left[\boldsymbol{\gamma}_{k}^{s}, \boldsymbol{\gamma}_{k}^{m}\right]^{T}$ represents parameters in system model and measurement model respectively. $\gamma_{k}$ belongs to the parameter domain $\mathcal{P} \subset \mathcal{R}^{K}$, where $K$ is the parameter dimension. The whole system is described by the following system equation

$$
\boldsymbol{x}_{k+1}=a_{k}\left(\boldsymbol{x}_{k}, \boldsymbol{\gamma}_{k}^{s}\right)+\boldsymbol{w}_{k},
$$

and measurement equation

$$
\boldsymbol{o}_{k+1}=h_{k}\left(\boldsymbol{x}_{k+1}, \boldsymbol{\gamma}_{k}^{m}\right)+\boldsymbol{v}_{k+1},
$$

where $a_{k}(\cdot)$ is the system function, which updates the current state to the next state. $h_{k}(\cdot)$ is the measurement function, which relates the state to the measurements. $\boldsymbol{w}_{k}$ and $\boldsymbol{v}_{k}$ represent system and measurement noise. The system can also be illustrated by a graphical model in Fig. 1 .

The Bayesian approach provides a recursive way to estimate the hidden state of dynamic systems with the above form. It has also two steps: prediction step

$$
f_{k+1}^{p}\left(\boldsymbol{x}_{k+1}\right)=\int_{\mathcal{L}} f_{k}^{T}\left(\boldsymbol{x}_{k+1} \mid \boldsymbol{x}_{k}, \gamma_{k}^{s}\right) f_{k}^{e}\left(\boldsymbol{x}_{k}\right) \mathrm{d} \boldsymbol{x}_{k}
$$

and update step

$$
f_{k+1}^{e}\left(\boldsymbol{x}_{k+1}\right)=\frac{1}{c_{k}} f_{k+1}^{L}\left(\boldsymbol{o}_{k+1} \mid \boldsymbol{x}_{k+1}, \gamma_{k}^{m}\right) f_{k+1}^{p}\left(\boldsymbol{x}_{k+1}\right)
$$

where $f_{k+1}^{p}\left(\boldsymbol{x}_{k+1}\right)$ is the predicted density at time $k$ $f_{k+1}^{T}\left(\boldsymbol{x}_{k+1} \mid \boldsymbol{x}_{k}, \boldsymbol{\gamma}_{k}^{s}\right)$ is the transition density, which is given by

$$
f_{k+1}^{T}\left(\boldsymbol{x}_{k+1} \mid \boldsymbol{x}_{k}, \boldsymbol{u}_{k}\right)=f_{k}^{w}\left(\boldsymbol{x}_{k+1}-a_{k}\left(\boldsymbol{x}_{k}, \gamma_{k}^{s}\right)\right),
$$

where $f_{k}^{w}(\cdot)$ is the density of the system noise at time $k$. $f_{k}^{e}\left(\boldsymbol{x}_{k}\right)$ is the posterior density function at time $k . c_{k}$

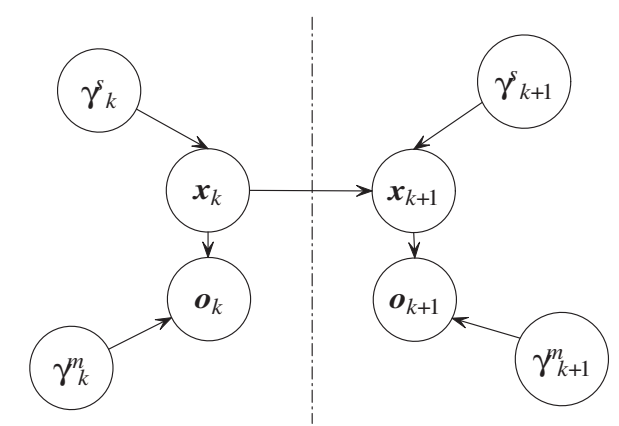

Fig. 1. A sensor fusion graphic model for localization problem. is the normalization constant. $f_{k+1}^{L}\left(\boldsymbol{y}_{k+1} \mid \boldsymbol{x}_{k+1}, \gamma_{k}^{m}\right)$ is the conditional likelihood density given by

$$
f_{k+1}^{L}\left(\boldsymbol{y}_{k+1} \mid \boldsymbol{x}_{k+1}\right)=f_{k+1}^{v}\left(\boldsymbol{y}_{k+1}-h_{k+1}\left(\boldsymbol{x}_{k+1}, \gamma_{k}^{m}\right)\right),
$$

where $f_{k}^{v}(\cdot)$ is the density of the measurement noise at time $k$.

If the parameters are not accurately known or vary with time, they can be also regarded as states. So Eq. (1) and Eq.(2) become

$$
\left[\begin{array}{c}
\boldsymbol{x}_{k+1} \\
\boldsymbol{\gamma}_{k+1}
\end{array}\right]=\left[\begin{array}{c}
a_{\boldsymbol{x}, k}\left(\boldsymbol{x}_{k}, \boldsymbol{\gamma}_{k}^{s}\right)+\boldsymbol{w}_{\boldsymbol{x}, k} \\
a_{\gamma, k}\left(\boldsymbol{\gamma}_{k}\right)+\boldsymbol{w}_{\gamma, k}
\end{array}\right]
$$

and

$$
\boldsymbol{o}_{k+1}=h_{k}\left(\boldsymbol{x}_{k+1}, \boldsymbol{\gamma}_{k}^{m}\right)+\boldsymbol{v}_{k+1} .
$$

The joint state and parameter probabilities can also be derived by Bayesian framework as the following

$$
\begin{aligned}
& f_{k+1}^{p}\left(\boldsymbol{x}_{k+1}, \gamma_{k+1}\right) \\
& =\int_{\mathcal{P}} \int_{\mathcal{L}} f_{k}^{T}\left(\boldsymbol{x}_{k+1}, \gamma_{k+1} \mid \boldsymbol{x}_{k}, \gamma_{k}\right) f_{k}^{e}\left(\boldsymbol{x}_{k}, \boldsymbol{\gamma}_{k}\right) \mathrm{d} \boldsymbol{x}_{k} \mathrm{~d} \boldsymbol{\gamma}_{k},
\end{aligned}
$$

and

$$
\begin{aligned}
& f_{k+1}^{e}\left(\boldsymbol{x}_{k+1}, \boldsymbol{\gamma}_{k+1}\right) \\
& =\frac{1}{c_{k}} f_{k+1}^{L}\left(\boldsymbol{o}_{k+1} \mid \boldsymbol{x}_{k+1}, \boldsymbol{\gamma}_{k+1}\right) f_{k+1}^{p}\left(\boldsymbol{x}_{k+1}, \boldsymbol{\gamma}_{k+1}\right)
\end{aligned}
$$

Afterwards, the marginal posterior state and parameter densities are easily obtained by

$$
f_{k}^{e}\left(\boldsymbol{x}_{k}\right)=\int_{\mathcal{P}} f_{k}^{e}\left(\boldsymbol{x}_{k}, \gamma_{k}\right) \mathrm{d} \gamma_{k}
$$

and

$$
f_{k}^{e}\left(\gamma_{k}\right)=\int_{\mathcal{L}} f_{k}^{e}\left(\boldsymbol{x}_{k}, \gamma_{k}\right) \mathrm{d} \boldsymbol{x}_{k}
$$

The above equations provide a general framework for simultaneous state and parameter estimation problem. Theoretically, many problems in the localization area can be solved by this framework, such as dynamical radio map estimation, sensor bias estimation, motion parameter estimation and so on. In the following, we use this framework to simultaneously fuse the RSS measurements, pressure measurements and a topological map, and estimate the spatial and time varying bias for pressure measurement. In this example application, one advantage is that the states are estimated by two different information sources but the unknown parameter is only related to one of them. So different from state and parameter estimation algorithms, the two information sources can teach each other to ensure the convergence and robustness of the final result.

\section{Models OF INFORMATION SOURCES}

\section{A. RSS of WLAN signal}

In WLAN based localization systems, RSS is most often used as the input of the positioning algorithm because it is much easier to obtain than the time or the angle information. The relation between the location and the RSS is modeled 
by the so-called radio map function $R:=R(\boldsymbol{x})$, where $\boldsymbol{x}=$ $[x, y, z]^{T}$ is the 3-D location vector. In theory, the radio map function follows the radio propagation rule. But in reality, due to the complexity of indoor environment, the radio map function has a very complicated form. As illustrated in Fig. 2 , a real measured radio map in a office building is hard to be described in an analytic way. So in practice, the radio map function is usually modeled in a non-parametric way by a number of selected grid points, i.e., $\boldsymbol{x} \in\left[x^{i}, y^{i}, z^{i}\right]^{T}$, where $i=1,2, \ldots, M$ is the index of grid points. In addition, since the wireless channel is influenced by many factors, e.g., measurement noise, changing environment, and moving people, the measured signal power fluctuates with time as shown in Fig. 3, i.e., $r=R(\boldsymbol{x})+\triangle(\boldsymbol{x}, t)+w$, where $\triangle(\boldsymbol{x}, t)$ could be regarded as an unknown spatial and time varying parameter indicating the inaccuracy of radio map model and its temporal variation; $w$ is the measurement noise, usually regarded as a Gaussian as Fig. 3. In this paper, for simplicity, we assume a non-parametric radio map model that is accurately known and time invariant. Then the measurement equation for the RSS measurement from $\operatorname{AP} n$ becomes

$$
r^{n}=R^{n}(\boldsymbol{x})+w^{n}, \boldsymbol{x} \in\left[x^{i}, y^{i}, z^{i}\right]^{T}, i=1,2, \ldots, M .
$$

\section{B. Pressure Measurement From MEMS Barometric Sensor}

As it is well known, the atmospheric pressure is a physical property strongly related to the altitude. Assuming a constant temperature gradient of $\mathrm{d} T / \mathrm{d} z$, the altitude $z$ can be expressed as a function of pressure $p$ using the following standard equation,

$$
z=T_{0} /(-\mathrm{d} T / \mathrm{d} z) \cdot\left[1-\left(\frac{p}{p_{0}}\right)^{-\mathrm{d} T / \mathrm{d} z \cdot R / g}\right],
$$

where $T_{0}=288.15 \mathrm{~K}$ and $p_{0}=101325 \mathrm{~Pa}$ are the reference temperature and pressure, respectively. $R$ is in standard conditions equal to $287.052 \mathrm{~m}^{2} / \mathrm{s}^{2} / K ; g$ is equal to $9.82 \mathrm{~m} / \mathrm{s}^{2}$. If the change in pressure $\frac{\mathrm{d} p}{p_{0}}$ and temperature $\frac{\mathrm{d} T}{T}$ are small, the above equation can be approximated as

$$
\mathrm{d} z=-\frac{\mathrm{d} p \cdot R \cdot T}{g \cdot p_{0}}
$$
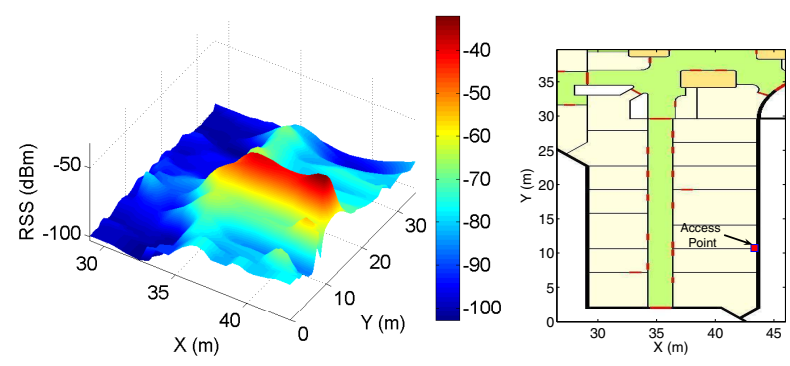

Fig. 2. A radio map example in an office building.
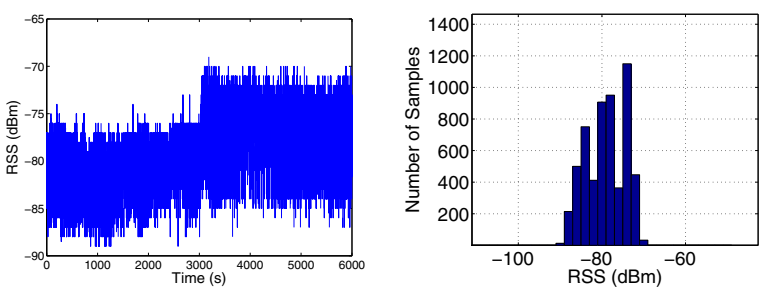

Fig. 3. Temporal variation and histogram of RSS measurements in 6000 seconds.

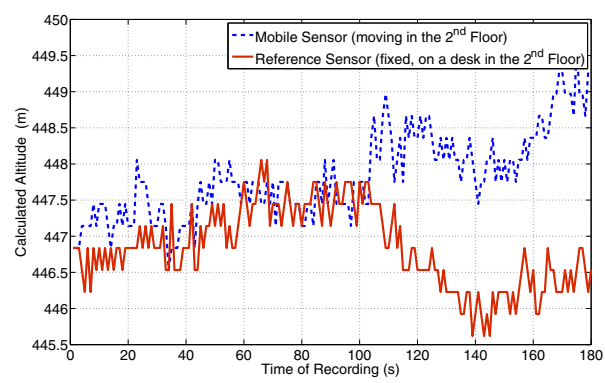

Fig. 4. Estimated altitudes by a static and a mobile barometric sensors.

which can be further modified to a linear function between $p$ and $z$ as

$$
p=\underbrace{\left(-\frac{p_{o} \cdot g}{T \cdot R}\right)}_{=\alpha} \cdot z+\underbrace{p^{\prime}+\frac{p_{o} \cdot g \cdot z^{\prime}}{T \cdot R}}_{=\beta},
$$

where $p^{\prime}$ and $z^{\prime}$ are the reference altitude and pressure. Fig. 4 shows the estimated altitude using pressure measurements by a static barometric sensor and a mobile barometric sensor, which moves in the same floor. We notice that the pressure is varying both temporally and spatially. Fortunately, when we consider the tracking problem, the pressure variation caused by horizontal moving and temporal environmental change is relatively small and slow. So we can simply assume that the pressure is only related to the height and a bias $\beta(t)$, which is slowly varying, i.e.,

$$
p(t)=\alpha \cdot z+\beta(t) .
$$

\section{Building Map}

A building map is another very important information source. The positions of obstacles, such as walls or doors, determine the possible routes where people can move. Mathematically, the map influences the prior joint probability of $f(x, y, z)$ and the transition density $f^{T}\left(\boldsymbol{x}_{k+1} \mid \boldsymbol{x}_{k}\right)$.

In this paper, we represent the building map by a topological graph consisting of a number of location points as shown in Fig. 5. These points are connected by their spatial relation, i.e., points which can see each other are connected. In this way, the indoor environmental restriction such as walls, doors or floors can be easily integrated. In addition, the topological graph can reflect the restriction to the location, e.g., the height $z$ is limited to discrete floor heights except in some special places such as stairs or elevators. With the topological 


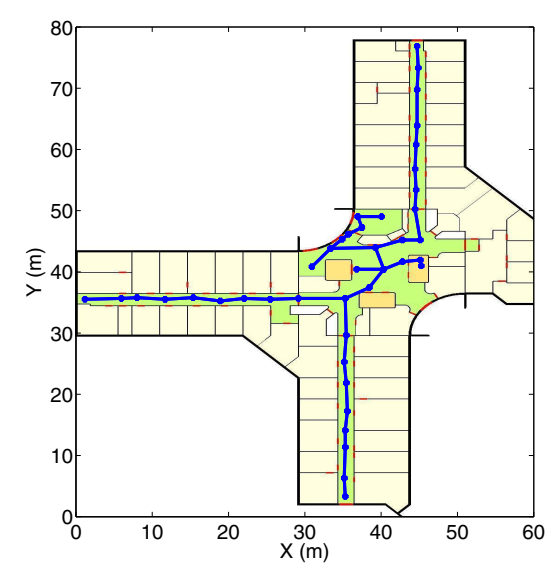

Fig. 5. Example of a topological map.

graph, the location transition density can be represented by a Gaussian distribution

$$
f^{T}\left(\boldsymbol{x}_{k+1}^{i} \mid \boldsymbol{x}_{k}^{j}\right)=\mathcal{N}\left(d_{s}(i, j)-\bar{v} \cdot \Delta t,\left(\sigma_{v} \cdot \Delta t\right)^{2}\right),
$$

where $d_{s}(i, j)$ is the shortest distance between $\boldsymbol{x}^{i}$ and $\boldsymbol{x}^{j}$, which can be calculated offline by Floyd's algorithm [13]. $\bar{v}$ is the mean of moving speed and $\sigma_{v}$ is the standard deviation of moving speed.

\section{BAYESIAN Filtering FOR SiMUlTANEOUS LOCALIZATION AND BIAS ESTIMATION}

In this section, the Bayesian framework are used to fuse all the information sources in the last section and simultaneously estimate the parameter $\beta(t)$. This is illustrated by a graph model in Fig. 6. The system is described by the following system function

$$
\left[\begin{array}{c}
\boldsymbol{x}_{k+1} \\
\beta_{k+1}
\end{array}\right]=\left[\begin{array}{c}
a\left(\boldsymbol{x}_{k}, m\right)+\boldsymbol{w}_{\boldsymbol{x}, k} \\
\beta_{k}+w_{\beta, k}
\end{array}\right]
$$

and measurement function

$$
\left[\begin{array}{l}
r_{k+1}^{n} \\
p_{k+1}
\end{array}\right]=\left[\begin{array}{c}
R^{n}\left(\boldsymbol{x}_{k+1}\right)+v_{r, k+1}^{n} \\
\alpha \cdot z_{k+1}+\beta_{k+1}+v_{p, k+1}
\end{array}\right],
$$

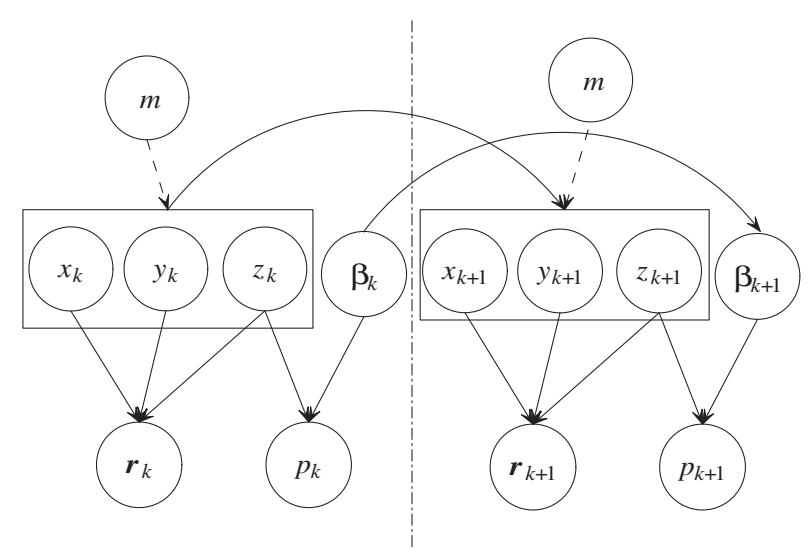

Fig. 6. A graphic model for simultaneous multi-information fusion and pressure bias estimation. where $m$ represents the information from the building map; $n=1 \ldots N$ is the index of AP; $w_{\beta}, v_{r}$ and $v_{p}$ are the noise terms for parameter prediction, RSS and pressure measurements, following Gaussian distribution $\mathcal{N}\left(0, \sigma_{\beta}\right), \mathcal{N}\left(0, \sigma_{r}\right)$ and $\mathcal{N}\left(0, \sigma_{p}\right)$ respectively. The corresponding posterior joint density function is hybrid, which includes both a discrete state $\boldsymbol{x}_{k}^{i}$ and a continuous state $\beta_{k}$. This can be derived as

$$
\begin{aligned}
& f_{k+1}^{p}\left(\boldsymbol{x}_{k+1}^{i}, \beta_{k+1}\right) \\
& =\sum_{j} \int_{\mathcal{R}} f_{k}^{T}\left(\boldsymbol{x}_{k+1}^{i}, \beta_{k+1} \mid \boldsymbol{x}_{k}^{j}, \beta_{k}, m\right) f_{k}^{e}\left(\boldsymbol{x}_{k}^{j}, \beta_{k}\right) \mathrm{d} \beta_{k}
\end{aligned}
$$

and

$$
\begin{aligned}
& f_{k+1}^{e}\left(\boldsymbol{x}_{k+1}^{i}, \beta_{k+1}\right) \\
& =\frac{1}{c_{k}} f_{k+1}^{L}\left(\boldsymbol{r}_{k+1}, b_{k+1} \mid \boldsymbol{x}_{k+1}^{i}, \beta_{k+1}\right) f_{k+1}^{p}\left(\boldsymbol{x}_{k+1}^{i}, \beta_{k+1}\right) .
\end{aligned}
$$

Combining (18), (19) and (20),

$$
\begin{aligned}
& f_{k+1}^{T}\left(\boldsymbol{x}_{k+1}^{i}, \beta_{k+1} \mid \boldsymbol{x}_{k}^{j}, \beta_{k}, m\right) \\
& =f_{\boldsymbol{x}^{i}, k}^{T}\left(\boldsymbol{x}_{k+1}^{i} \mid \boldsymbol{x}_{k}^{j}, m\right) f_{\beta, k}^{T}\left(\beta_{k+1} \mid \beta_{k}\right) \\
& =\mathcal{N}\left(d_{s}(i, j)-\bar{v}, \sigma_{v}^{2}\right) \cdot \mathcal{N}\left(\beta_{k+1}-\beta_{k}, \sigma_{\beta}^{2}\right),
\end{aligned}
$$

and

$$
\begin{aligned}
& f_{k+1}^{L}\left(\boldsymbol{r}_{k+1}, p_{k+1} \mid \boldsymbol{x}_{k+1}^{i}, \beta_{k+1}\right) \\
& =f_{\boldsymbol{r}, k+1}^{L}\left(\boldsymbol{r}_{k+1} \mid \boldsymbol{x}_{k+1}^{i}\right) f_{p, k+1}^{L}\left(p_{k+1} \mid z_{k+1}^{i}, \beta_{k+1}\right) \\
& =\prod_{n=1}^{N} \mathcal{N}\left(r^{n}-R^{n}\left(\boldsymbol{x}_{k+1}^{i}\right), \sigma_{r}^{2}\right) \\
& \cdot \mathcal{N}\left(p_{k+1}-\alpha \cdot z_{k+1}^{i}-\beta_{k+1}, \sigma_{p}^{2}\right)
\end{aligned}
$$

Given the uniformly or Gaussian distributed initial joint probability density $f_{k+1}\left(\boldsymbol{x}_{0}^{i}, \beta_{0}\right)$, the hybrid posterior density $f_{k}^{e}\left(\boldsymbol{x}_{k}^{i}, \beta_{k}\right)$ and $f_{k}^{e}\left(\beta_{k}\right)$ both have Gaussian mixture form. For each recursive step, the number of mixture components increases so that the optimal analytical solution is not tractable. The algorithm proposed in [14] can optimally reduce the number of Gaussian components and get the suboptimal solution. Besides, particle filters are also used by some researchers to solve the similar problem. One problem using particle filters is that when the parameter is part of state, the augmented state space model is not ergodic, and the uniform convergence result does not hold anymore [15].

In this paper, we use another suboptimal algorithm, socalled dual estimation [10] [16]. The idea of dual estimation is to separate joint state and parameter estimation into two independent processes. As illustrated in Fig. 7, the grid based filter is used to estimate the discrete location vector $\boldsymbol{x}_{k}$ assuming the parameter $\beta_{k}$ is known. The expectation of posterior $\boldsymbol{x}_{k}$ is sent back to the parameter estimator, which is a Kalman filter in our case. Afterwards, the estimated $\beta_{k}$ is sent again to the state estimator. The dual estimation at time $k$ stops either after a given number of iterations or if the new state estimation is close enough to the old one. Note 


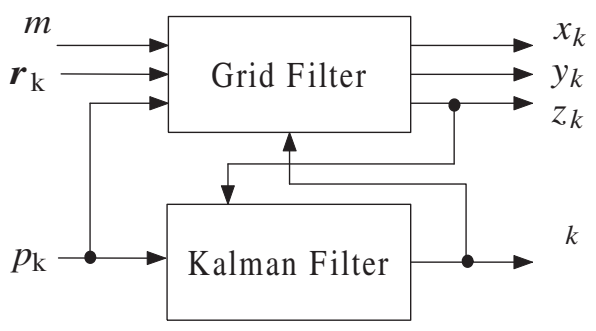

Fig. 7. Illustration for dual estimation.
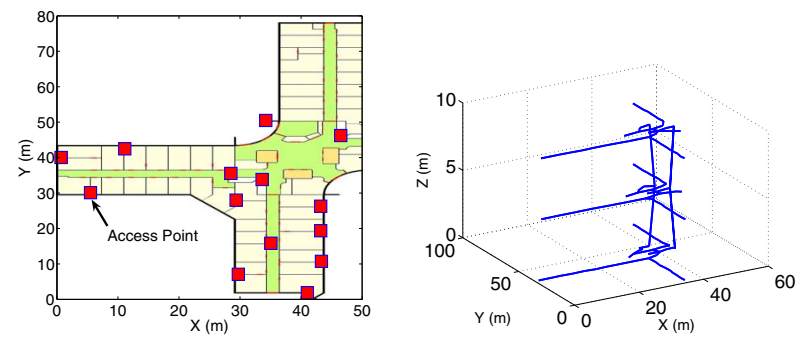

Fig. 8. Test environment and its topological graph for three floors.

that the dual estimation can be regarded as a generalized EM algorithm. Its convergence to the suboptimal solution is guaranteed by its iterative optimization process [16].

Since only the posterior expectations of height and sensor bias instead of the whole probability are exchanged between two filters, the dual estimation might bring a large error if the posterior density function has a complicated form. But in our case in most of the time the height $z$ is limited to the discrete floor height, the posterior of $z$ tends to be unimodal. The pressure bias has also a Gaussian-like shape. That assures the suboptimal dual estimation works well for our application.

\section{EXPERIMENT RESULTS}

\section{A. Experiment Setup}

We evaluate our algorithm in a typical multi-floor office building depicted in Fig. 5. Each floor has a area of $50 m \times 80 m$ and has a similar structure like the left figure in in Fig. 8. There are 14 access points installed on the first floor. We take three floors for evaluation. Since APs only exist on the first floor, the 3-D localization error could be large when users stay in the higher floors. A topological graph is built automatically based on the chosen reference points. Here we only take the points along the corridor, stairs, and elevator like the graph in Fig. 6. These points represent the basic moving possibilities. The rooms can also be easily added to the graph if some points in rooms are taken. The whole topological graph for three floors is shown in Fig 8. The RSS values at reference points are measured offline and their noise parameters are also estimated according to the measurements. In online step, we first walked in the corridor of the first floor and then went up to the second floor by stairs, walked around in the second floor and finally went up to the third floor by elevator. While moving, the RSS
TABLE I

The VAlues of PARAMETERS

\begin{tabular}{|c|c|}
\hline Parameter & Value \\
\hline$\sigma_{r}$ & $4 \mathrm{~dB}$ \\
\hline $\bar{v}$ & $0 \mathrm{~m} / \mathrm{s}$ \\
\hline$\sigma_{v}$ & $2 \mathrm{~m} / \mathrm{s}$ \\
\hline$\sigma_{p}$ & $6 \mathrm{~Pa}$ \\
\hline$\sigma_{\beta}$ & $3 \mathrm{~Pa}$ \\
\hline
\end{tabular}

TABLE II

3-D LOCALIZATION RESUlTS

\begin{tabular}{|c|c|c|c|}
\hline & NN & Fusion & $\begin{array}{c}\text { Fusion and } \\
\text { Bias Estimation }\end{array}$ \\
\hline Mean of 3-D Error (m) & 8.2 & 6.5 & 6.1 \\
\hline Standard Deviation of 3-D Error (m) & 7.8 & 6.6 & 6.3 \\
\hline Mean of Altitude Error (m) & 1.2 & 0.2 & 0 \\
\hline
\end{tabular}

and pressure measurements by the barometric sensor were recorded simultaneously.

\section{B. Results}

The parameters taken in our test are list in Table I. Table II shows the comparison of the nearest neighbour (NN) algorithm that only uses RSS measurements, the fusion algorithm (Fusion) that integrates the RSS, air pressure, and topological map using grid filter and an assumed sensor bias, and the algorithm that simultaneously estimates the location and parameter. The criteria for the comparison are the mean and standard deviation of 3-D localization errors as well as the mean altitude error. Fig. 9 plots the altitude error by different algorithms. Fig. 10 compares the true bias with estimated bias by simultaneous localization and bias estimation algorithm.

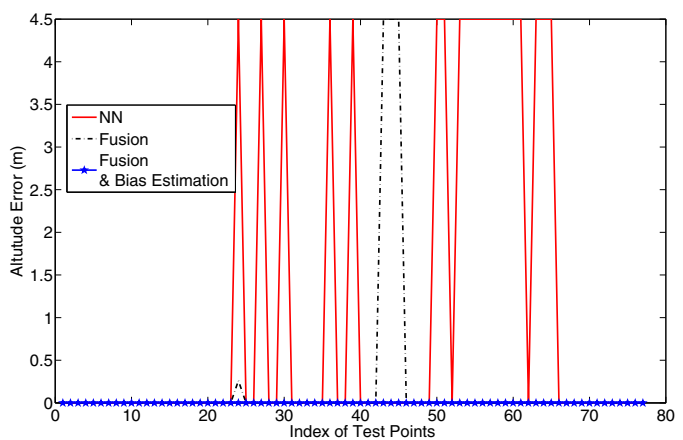

Fig. 9. Altitude error by different algorithms.

From Table II it can be seen that both the 2-D and the 3-D localization performances are improved by fusing more information sources. By simultaneously adapting the bias, the altitude error can be reduced to zero, i.e., perfect floor identification. 


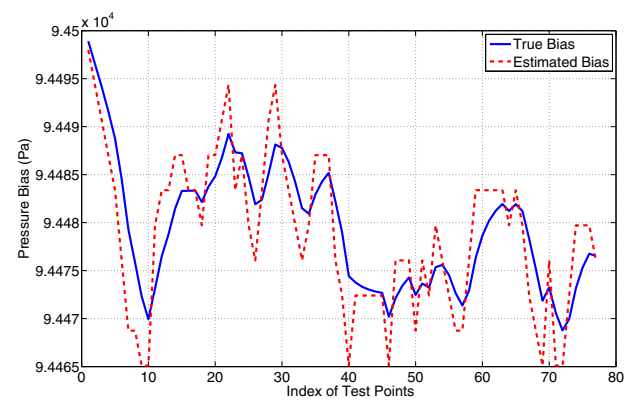

Fig. 10. Comparison between true bias and estimated bias by fusion and bias estimation. The true bias is obtained by filtering the difference between the measured pressures and calculated pressures.

\section{Sensitivity Analysis}

The algorithm in our previous paper [4] can also provide very good performance for floor identification. But since it is actually a self-calibration algorithm, the initial condition is very important. Given the wrong initial condition, the result can be totally wrong. The algorithm in this paper uses the RSS measurement to teach the bias estimation and hence make sure that the bias can be tracked without any limitation for initial value. To validate this, we add an artificial pressure bias $-60 P a$ to the real pressure measurement so that the wrong floor (10 meters higher) is identified if only depending on the pressure. Besides, we start with moving in the 3rd floor so that the intial RSS is also very unaccurate. The altitude errors are given in Fig. 11. We see that compare the result in Fig. 9, the error by fusion is obviously larger. That is because adding the artificial bias, the wrong floor is identified by pressure. If the same wrong floor also happened to be estimated by RSS measurements, the altitude error become bigger. But by simultaneously estimating the bias, the sensor bias can be quickly calibrated and then the altitude error becomes zero.

Additionally, we notice that the height $z$ is the variable that influences both RSS and pressure measurements. Through the inference of RSS and the correlation between $(x, y)$ and $z$, the estimated $z$ can be used to train the $\beta$ to ensure the convergence.

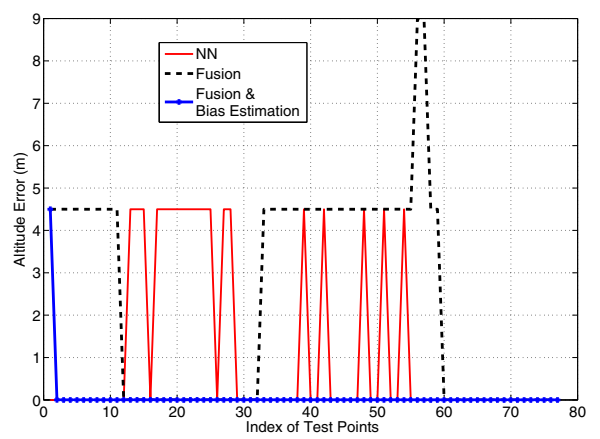

Fig. 11. Altitude error by different algorithms with an artificial bias.

\section{CONCLUSIONS AND FUTURE WORKS}

Bayesian framework can be used to solve joint state and parameter estimation problems in indoor positioning systems. In this paper, we apply this framework to a specific problem: simultaneous localization and sensor bias estimation. By fusing pressure measurements, a topological graph with RSS measurements and simultaneously estimating the pressure bias, the WLAN indoor positioning system becomes more robust and more accurate. In the next step, we will investigate the feasibility of using Bayesian framework for more complicated parameters, such as the parameter for the radio map generation and other state and parameter estimation problems in indoor positioning systems. Other more complicated filtering techniques like Gaussian mixture filter or particle filter will also be considered to solve the joint density estimation problems with proper forms.

\section{REFERENCES}

[1] P. Bahland V. N. Padmanabhan, "RADAR: An in-building RF-based user location and tracking system," in IEEE INFOCOM 2000, pp.775784.

[2] T. Roos, P. Myllymaki, H. Tirri, P. Misikangas, and J. Sievanen, "A probabilistic approach to WLAN user location estimation," International Journal of Wireless Information Networks, vlo. 9, no. 3, 2002.

[3] H. Lenz, B. B. Parodi, H. Wang, A. Szabo, J. Bamberger, J. Horn, and U. D. Hanebeck, "Adaptive Localization in Adaptive Networks", Chapter of Signal Processing Techniques for Knowledge Extraction and Information Fusion. Springer, 2008.

[4] H. Wang, H. Lenz, A. Szabo, U. D. Hanebeck, J. Bamberger, "Fusion of Barometric Sensors, WLAN Signals and Building Information for 3-D Indoor Campus Localization", in Proceedings of International Conference on Multisensor Fusion and Integration for Intelligent Systems (MFI 2006), pp. 426-432, Heidelberg, Germany, 2006.

[5] H. Wang, H. Lenz, A. Szabo, J. Bamberger, U. D. Hanebeck, "WLANBased Pedestrian Tracking Using Particle Filters and Low-Cost MEMS Sensors", in Proceedings of 4th Workshop on Positioning, Navigation and Communication 2007 (WPNC'07), Hannover, Germany, 2007.

[6] H.W. Sorenson, Kalman Filtering: Theory and Application. Piscataway, NJ: IEEE, 1985.

[7] S. J. Julier and J. K. Uhlmann, "Unscented Filtering and Nonlinear Estimation," in Proceedings of the IEEE, vol. 92, no. 3, 2004.

[8] B. Ristic, S. Arulamplalm, N. Gordon, Beyond the Kalman filter. Artech House, Boston, 2004.

[9] M. F. Huber and U. D. Hanebeck, "The Hybrid Density Filter for Nonlinear Estimation based on Hybrid Conditional Density Approximation," in Proceeding of the Tenth International Conference on Information Fusion (FUSION), 2007.

[10] E. Wan and A. Nelson, "Dual extended Kalman filter methods," in Kalman Filtering and Neural Networks (chap. 5), S. Haykin Ed. New York: Wiley, 2001.

[11] A. Dempster, N. Laird, and D. Rubin. "Maximum likelihood from incomplete data via the EM algorithm," Journal of the Royal Statistical Society, Series B, 39(1):1-38, 1977.

[12] H. Durrant-Whyte, and T. Bailey, "Simultaneous Localisation and Mapping (SLAM): Part I The Essential Algorithms," Robotics and Automation Magazine 13: 99-110.

[13] R. W. Floyd, "Algorithm 97: Shortest Path". Communications of the ACM 5 (6): 345.1962.

[14] U. D. Hanebeck, O. Feiermann, "Progressive Bayesian Estimation for Nonlinear Discrete-Time Systems: The Filter Step for Scalar Measurements and Multidimensional States", in Proceedings of the 2003 IEEE Conference on Decision and Control (CDC 2003), pp. 5366-5371, Maui, Hawaii, December, 2003.

[15] D. Crisan, J. Gaines, T. Lyons, "Convergence of a branching particle method to the solution of the Zakai equation," SIAM J. Appl. Math., vol. 58, no. 5, pp. 1568-1598, 1998.

[16] Z. Chen, "Bayesian filtering: from Kalman filters to particle filters, and beyond," Technical Report, McMaster University, 2006 